\title{
A salinomicina para o controle da eimeriose de caprinos leiteiros nas fases de cria e recria
}

\author{
The salinomicin in the control of eimeriosis in dairy goats at pre \\ and post-weaning phases
}

\author{
Luiz da Silva Vieira ${ }^{1^{*}}$ Nelson Nogueira Barros ${ }^{2}$ Antônio César Rocha Cavalcante ${ }^{3}$ \\ Luciano Jany Feijão Ximenes ${ }^{4}$ Rubênio Borges de Carvalho ${ }^{5}$
}

\section{RESUMO}

A salinomicina foi avaliada no controle da eimeriose caprina em 27 cabritos mestiços distribuídos aleatoriamente em três tratamentos num delineamento inteiramente casualizado: T0, não medicados (grupo controle); $T 1$ e T2, medicados com doses de 1 e $2 \mathrm{mg}$ de salinomicina/kg de peso vivo/dia, respectivamente. Na fase de cria, não houve diferença estatística $(P>0,005)$ no ganho médio de peso (GP) entre os três tratamentos. Na fase de recria, $o$ grupo T0 apresentou GP significativamente inferior $(P<0,01)$ aos grupos T1 e T2 entre estes não houve diferença significativa $(P>0,005)$. O número médio de oocistos por grama de fezes (OOPG) do grupo TO nas duas fases estudadas, foi significativamente maior $(P<0,01)$ ao dos grupos $T 1$ e T2; sendo que os grupos tratados não diferiram $(P>0,005)$ entre si. $O$ grupo T0 apresentou rendimento médio de carcaça $(R C)$ significativamente inferior $(P<0,05)$ aos grupos T1 e T2; entre estes não houve diferença significativa $(P>0,005)$. O peso médio da massa corporal $(M C)$ do grupo T0 foi inferior $(P<0,05)$ ao do grupo T2 e, não diferiu $(P>0,005)$ do grupo T1; entre os grupos T1 e T2, não houve diferença significativa $(P>0,005)$ no peso médio da massa corporal (MC). O uso da salinomicina nas doses de 1 e $2 \mathrm{mg} / \mathrm{kg}$, resultou em maior ganho de peso dos animais dos grupos T1 e T2 e, consequentemente, maior valor da margem bruta destes tratamentos. Os resultados obtidos mostraram que os $T 1$ e T2 foram equivalentes para o controle da eimeriose caprina, uma vez que ambos os tratamentos apresentaram maior ganho de peso e oocistograma inferior ao grupo T0. Conclui-se que o tratamento com a salinomicina na dose de 1,0mg/kg é eficaz, desde que seja administrada a partir da segunda semana de vida.

Palavras-chave: caprinos, Eimeria, controle, quimioterapia, antibiótico ionóforo, salinomicina.

\section{ABSTRACT}

Salinomicin was evaluated for the control of caprine eimeriosis in the pre-weaning (PRW) and post-weaning (POW) phases. Twenty seven crossbred goat kids were randomly distributed among three treatments: T0, not medicated (check group); $T$ and $T$ daily medicated with 1.0 and $2.0 \mathrm{mg} / \mathrm{kg}$ living weight of sålinomicin, respectively. In the PRW, there were not significant differences $(P>0.05)$ in the daily weight gain among the treatments. However, in the POW, the TO group presented daily weight gain inferior $(P>0.05)$ to the T1 and T2 groups, which did not statiscally differ $(P>0.05)$ between themselves. The average number of oocistes per gram of faeces in the TO at the two studied phases was significatively higher $(P<0.01)$ than in the T1 and T2, which did not statiscally differ $(P>0.05)$ between themselves. The average carcass yield was significantly lower $(P<0.05)$ in the T0 group than in the $T 2$, which presented similar results $(P>0.05)$ to the $T 1$ group. The use of salinomicin resulted in higher weight gains by the lambs, and improved the economic yield. The treatments T1 and $T 2$ were equivalent in the caprine eimeriosis control since both treatments presented higher weight gains and lower oocistogram than the treatment TO. However, treatment T1 should be recommended due to its better economic efficiency.

Key words: goats, Eimeria, control, quimioterapy, salinomicin.

\section{INTRODUÇÃO}

A eimeriose ou coccidiose caprina é uma doença infecciosa causada por protozoários coccídicos do gênero Eimeria, que acomete principalmente caprinos jovens. É uma parasitose de distribuição mundial, atingindo caprinos submetidos aos mais diferentes sistemas de manejo, embora seja mais grave e mais freqüente em animais criados em sistemas intensivos, daí a sua importância em rebanhos leiteiros (REBOUÇAS et al., 1992; SUAREZ, 1995).

O controle da eimeriose dos caprinos deve ser realizado através de práticas adequadas de manejo

${ }^{1}$ Médico Veterinário, Doutor, Embrapa Caprinos, CP D-10, 62011-970, Sobral, CE. E-mail: lvieira@ cnpc.embrapa.br. Autor para correspondência

${ }^{2}$ Médico Veterinário, MSc, Embrapa Caprinos.

${ }^{3}$ Médico Veterinário, MSc, Embrapa Caprinos.

${ }^{4}$ Zootecnista, Convênio Universidade Estadual Vale do Acaraú - UVA/Embrapa Caprinos.

${ }^{5}$ Economista, MSc. 
e pela administração de quimioterápicos com o objetivo de impedir ou reduzir a infecção que ocorre através da ingestão de oocistos esporulados na água e na ração contaminadas. A utilização de drogas específicas contra a eimeriose é de valor relativo quando já existem manifestações clínicas, isto porque já houve destruição de tecidos e as drogas não são capazes de regenerá-los. Além disso, geralmente os coccidiostáticos atuam apenas nas fases precoces de multiplicação dos parasitos, não agindo, portanto, nas formas sexuadas que geralmente são as mais patogênicas. Desta forma, o tratamento preventivo em todo o rebanho susceptível (animais jovens) iniciado logo após a exposição dos cabritos às formas infectivas, será mais eficaz do que o tratamento curativo. A salinomicina é um antibiótico ionóforo que tem sido eficiente no controle da eimeriose em bovinos e ovinos (LIMA et al., 1985; FACURY FILHO, 1992) entretanto, trabalhos experimentais relativos à eficácia desses compostos em caprinos são bastante limitados. OLIVEIRA et al., (1997) verificaram em caprinos que a salinomicina na dose de $1 \mathrm{mg} / \mathrm{kg}$, administrada continuamente no suplemento mineral, no período do nascimento até a desmama, reduziu significativamente o número de oocistos por grama de fezes (OOPG), embora não tenha promovido aumento significativo no peso dos animais. Por outro lado, a salinomicina em doses elevadas pode provocar intoxicação, uma vez que possui radicais ionóforos, que agem na membrana celular (SANTA ROSA \& PINHEIRO, 1996). Esses autores verificaram sinais clínicos de intoxicação e mortalidade em caprinos medicados com salinomicina na dose de $2 \mathrm{mg} / \mathrm{kg}$, adcionada ao leite e administrada em grupos duas vezes por dia (manhã e tarde). Os sinais clínicos surgiram 24 horas após o tratamento e se caracterizaram por dispnéia e letargia, sendo que os casos de mortalidade ocorreram entre 12 e 48 horas após o aparecimento dos sinais. Na necrópsia foram constadas lesões na musculatura cardíaca e em menor proporção nos rins.

Este trabalho foi realizado com o objetivo de avaliar um programa de controle da eimeriose em caprinos leiteiros através do uso preventivo de salinomicina nas fases de cria e recria.

\section{MATERIAL E MÉTODOS}

Este trabalho foi realizado na área experimental da Embrapa Caprinos em Sobral-CE. As crias foram separadas das mães imediatamente após o nascimento e alimentadas artificialmente nos dois primeiros dias de vida com colostro de cabra submetido à temperatura de $56^{\circ} \mathrm{C}$, durante uma hora. A partir de dois dias de nascidos e durante a fase de cria, que durou 63 dias, os cabritos foram alimentados artificialmente com leite de vaca pasteurizado. Até a primeira semana de idade, os cabritos foram mantidos em uma baia próxima a das mães, para possibilitar a ocorrência de infecção natural por espécies do gênero Eimeria.

Foram utilizados 27 cabritos machos, mestiços (1/2 Pardo alpino x 1/2 Moxotó), os quais foram pesados e distribuídos aleatoriamente em três tratamentos, T0, T1 e T2, num delineamento experimental inteiramente casualizado. No T0, os animais não foram medicados (grupo controle), no T1 e T2 foram medicados diariamente com salinomicina nas doses de $1 \mathrm{mg} /$ $\mathrm{kg} \mathrm{e} 2 \mathrm{mg} / \mathrm{kg}$, respectivamente.

Os cabritos foram introduzidos no experimento com uma semana de vida, sendo acompanhados até os 126 dias de idade. Um mês após o início do experimento, observou-se que, apesar da exposição natural, o nível de infecção dos animais do grupo controle não havia alcançado um oocistograma médio de 1.000 oocistos por grama de fezes (OOPG). Da mesma forma, o OOPG dos grupos T1 e T2 no primeiro mês do experimento, permaneceu nulo ou muito baixo, não ultrapassando 200 oocistos por grama de fezes, razão pela qual todos os animais experimentais foram infectados via oral, com aproximadamente 500 oocistos de Eimeria spp.

Durante a fase de cria, os cabritos foram mantidos em gaiolas individuais e alimentados artificialmente com leite de vaca pasteurizado oferecido na proporção de $20 \%$ do peso corporal/dia, dividido em duas mamadas (manhã e tarde) de igual volume. A salinomicina nesta fase foi administrada individualmente junto com o leite oferecido pela manhã. Todos os animais foram pesados ao nascer e em seguida semanalmente, ocasião em que era feito o reajuste do leite e de salinomicina. A partir da sexta semana de vida, a quantidade de leite não foi reajustada, entretanto, o reajuste da salionomicina foi realizado até o final do experimento. A partir do décimo quinto dia de vida, todos os animais receberam feno de leucena, capim elefante picado e concentrado com $19 \%$ de proteína bruta e 78\% de NDT, "ad libitum. Os cabritos foram desaleitados aos 63 dias de idade, quando então foi finalizada a fase de cria, dando-se início à segunda fase do experimento, a recria.

Durante a fase de recria os cabritos permaneceram em baias coletivas, sendo utilizada uma baia para cada tratamento. Nesta fase, os animais experimentais receberam um concentrado contendo $16 \%$ de proteína bruta e $75 \%$ de NDT, que foi oferecido na proporção de $1,5 \%$ do peso vivo. Os cabritos receberam ainda feno de leucena, na proporção de $1 \%$ do 
peso vivo, além de capim elefante "ad libitum". No período da recria, a salinomicina foi diluída em água e fornecida em mamadeiras individuais, por períodos de 14 dias consecutivos, com intervalos de igual tamanho, até os 126 dias de idade, quando terminou o experimento. As pesagens na fase de recria foram efetuadas ao desmame e aos 70, 84, 98, 112 e 126 dias de idade. A cada 14 dias, foram realizadas coleta de amostras de fezes, em todos os animais. Cada amostra foi submetida à contagem de OOPG de acordo com a técnica descrita por UENO \& GONÇALVES (1988).

Foram estudados os seguintes parâmetros: ganho de peso médio diário nas fases de cria e recria e oocistograma médio nas duas fases de estudo. No final do experimento, todos os animais foram abatidos para determinação do rendimento de carcaça quente e massa corporal. Esta última constou da pesagem da pele, carcaça, patas, cabeça e vísceras, tendo-se esvaziado o trato digestivo, a bexiga e a vesícula biliar.

Os dados de ganho de peso, rendimento de carcaça e massa corporal foram submetidos à análise de variância e as comparações entre as médias foram feitas pelo teste de Tukey (PIMENTEL GOMES, 1977). Para a análise de variância e comparações entre as médias, os dados referentes ao OOPG foram transformados para $\log (\mathrm{OOPGx} 0,02+4,5)$, visando à uniformização da variância das médias. Foi procedida uma análise de correlação e outra de regressão entre as variáveis estudadas, utilizando-se os níveis de salinomicina como variável independente e o ganho de peso diário nas fases de cria (GPDC) e recria (GPDR), o oocistograma nas fases de cria (OOPGC) e recria, (OOPGR), o rendimento de carcaça $(\mathrm{RC})$ e a massa corporal (MC) como variáveis dependentes.

Foram levantados os custos e a receita, abrangendo as fases de cria e recria, bem como calcu- lada a margem bruta, para determinar o benefício gerado sobre o valor dos animais através do controle preventivo da eimeriose. A receita bruta foi gerada a partir do produto do ganho médio de peso e do preço de venda de caprinos no mercado, que corresponde a $\mathrm{R} \$$ $1,30 / \mathrm{kg}$ de peso vivo. Os custos foram estimados com base no arraçoamento dos animais, uso de medicamentos e na utilização de mão-de-obra. O uso de mão-deobra foi estimado em 1,5 horas/homem/dia, sendo o custo calculado com base no salário mínimo vigente ( R\$ 136,00), acrescido dos encargos sociais, totalizando $\mathrm{R} \$ 165,92$. As margens brutas encontradas foram submetidas a análise de variância, a 5\% de probalidade, para identificar diferença significativa ou não entre os níveis de salinomicina utilizados.

\section{RESULTADOS E DISCUSSÃO}

Na tabela 1, estão expressos os valores médios de ganho de peso, oocistograma (OOPG), rendimento de carcaça e massa corporal. $\mathrm{O}$ ganho de peso na fase de cria foi a única variável não influenciada pelos tratamentos, ou seja, as demais variáveis apresentaram comportamento linear.

Analisando a tabela 2, observa-se que o tratamento preventivo com a salinomicina nas dose de 1 e $2 \mathrm{mg} / \mathrm{kg}$ influenciou ( $>00,01)$ o ganho de peso somente na fase de recria. Este incremento no ganho de peso foi expressivo, $34,3 \mathrm{~g} / \mathrm{g}$ de salinomicina ingerida. É provável que OLIVEIRA et al.,(1997) não tenham observado incremento no ganho de peso em caprinos medicados preventivamente com a salinomicina na dose de $1 \mathrm{mg} / \mathrm{kg}$ porque os autores administraram o antibiótico ionóforo apenas até a fase de cria .No presente trabalho, na fase de cria também não houve diferença significativa $(\mathrm{p}<0,05)$ entre os grupos medica-

Tabela 1 - Valores médios ( \pm erro padrão) referentes ao ganho de peso, número de oocistos por grama de fezes (OOPG), rendimento de carcaça e massa corporal em caprinos submetidos ao tratamento preventivo com salinomicina, nas fases de cria e recria.

\begin{tabular}{|c|c|c|c|}
\hline \multirow{2}{*}{ Variáveis } & \multicolumn{3}{|c|}{ Tratamentos } \\
\hline & $\mathrm{T}_{0}$ & $\mathrm{~T}_{1}$ & $\mathrm{~T}_{2}$ \\
\hline \multicolumn{4}{|l|}{ Ganho de peso (g/dia) } \\
\hline Fase de cria & $100,21 \pm 5,2^{\mathrm{a}}$ & $105,1 \pm 5,2^{\mathrm{a}}$ & $111,4 \pm 5,2^{\mathrm{a}}$ \\
\hline Fase de recria & $46,9 \pm 10,1^{\mathrm{a}}$ & $103,8 \pm 10,1^{\mathrm{b}}$ & $118 \pm 10,1^{b}$ \\
\hline \multicolumn{4}{|l|}{ OOPG nas fezes } \\
\hline Fase de cria & $114 \pm 6318^{\mathrm{a}}$ & $344 \pm 189^{b}$ & $44 \pm 19^{b}$ \\
\hline Fase de recria & $28.209 \pm 6917^{\mathrm{a}}$ & $718 \pm 112^{\mathrm{b}}$ & $248 \pm 83^{b}$ \\
\hline Rendimento de carcaça (\%) & $42,6 \pm 0,7^{\mathrm{a}}$ & $46,6 \pm 0,7^{b}$ & $45,8 \pm 0,7^{\mathrm{b}}$ \\
\hline Massa corporal $(\mathrm{kg})$ & $10,2 \pm 0,6^{\mathrm{a}}$ & $11,6 \pm 0,6^{\mathrm{ab}}$ & $12,6 \pm 0,6^{\mathrm{b}}$ \\
\hline
\end{tabular}

${ }^{1}$ Valores que na mesma linha, forem seguidos de letras diferentes, diferem significativamente entre si $(\mathrm{P}<0,05)$ pelo teste de Tukey. 
Tabela 2 - Estimativas das regressões do efeito dos níveis de salinomicina sobre o ganho de peso diário nas fases de cria (GPDC) e recria (GPDR), oocistograma nas fases de cria (OOPGC) e recria, (OOPGR), rendimento de carcaça (RC) e massa corporal (MC) em caprinos.

\begin{tabular}{|c|c|c|c|c|}
\hline \multirow{2}{*}{ Variável } & \multicolumn{2}{|c|}{ Constante da regressão } & \multicolumn{2}{|c|}{ Variáveis explicativas } \\
\hline & & Coeficiente de regressão & $\mathrm{R}^{2}$ & Nível de significância \\
\hline GPDC & 0,10 & $+3,97 \mathrm{X}$ & 0.045 & NS \\
\hline GPDR & 0,05 & $+34,3 \mathrm{X}$ & 0,465 & $* *$ \\
\hline OOPGC & 9597,5 & $-5602,6 X$ & 0,163 & $*$ \\
\hline OOPGR & 33488,7 & $-13.688,4 X$ & 0,435 & $* *$ \\
\hline $\mathrm{RC}$ & 43,47 & $+1,34 \mathrm{X}$ & 0,173 & $*$ \\
\hline $\mathrm{MC}$ & 10,3 & $+1,15 X$ & 0,212 & $*$ \\
\hline
\end{tabular}

$\mathrm{NS}=\mathrm{P}>0,05, *=\mathrm{P}<0,05 \mathrm{e}^{* *}=\mathrm{P}<0,01$.

dos (T1 e T2) e o grupo controle (T0), confirmando os dados relatados por OLIVEIRA et al.,(1997) .

Com relação aos achados parasitológicos, o oocistograma decresceu $(\mathrm{p}<0,01)$ de forma linear na medida em que se aumentou o consumo de salinomicina na dieta dos animais. Para cada grama de salinomicina ingerida nas fases de cria e recria, o oocistograma sofreu uma redução respectivamente, de 5.602,6 e 13.688,4 oocistos (Tabela 2). Todavia, a influência da salinomicina na redução do oocistograma, tanto no T1 como no T2, foi mais acentuada na fase de recria (Tabela 2). As correlações entre o oocistograma e ganho de peso nas fases de cria e recria, foram respectiva- mente de $-0,33$ e $-0,71$, indicando que a redução no oocistograma resultou em incremento significativo nos ganho de peso dos animais dos grupos T1 e T2, sendo bem mais acentuado na fase de recria. De acordo com AUGUSTINE et al., (1987), os antibióticos ionóforos atuam sobre as fases precoces do ciclo de vida da Eimeria, reduzindo o desenvolvimento das formas assexuadas intracelulares. OLIVEIRA et al., (1997) também observaram redução de OOPG em caprinos medicados continuamente com salinomicina na dose de $1 \mathrm{mg} / \mathrm{kg}$, durante a fase de cria. Apesar do oocistograma dos animais do grupo controle ter atingido 61.811 oocistos por grama de fezes (Figura 1) no

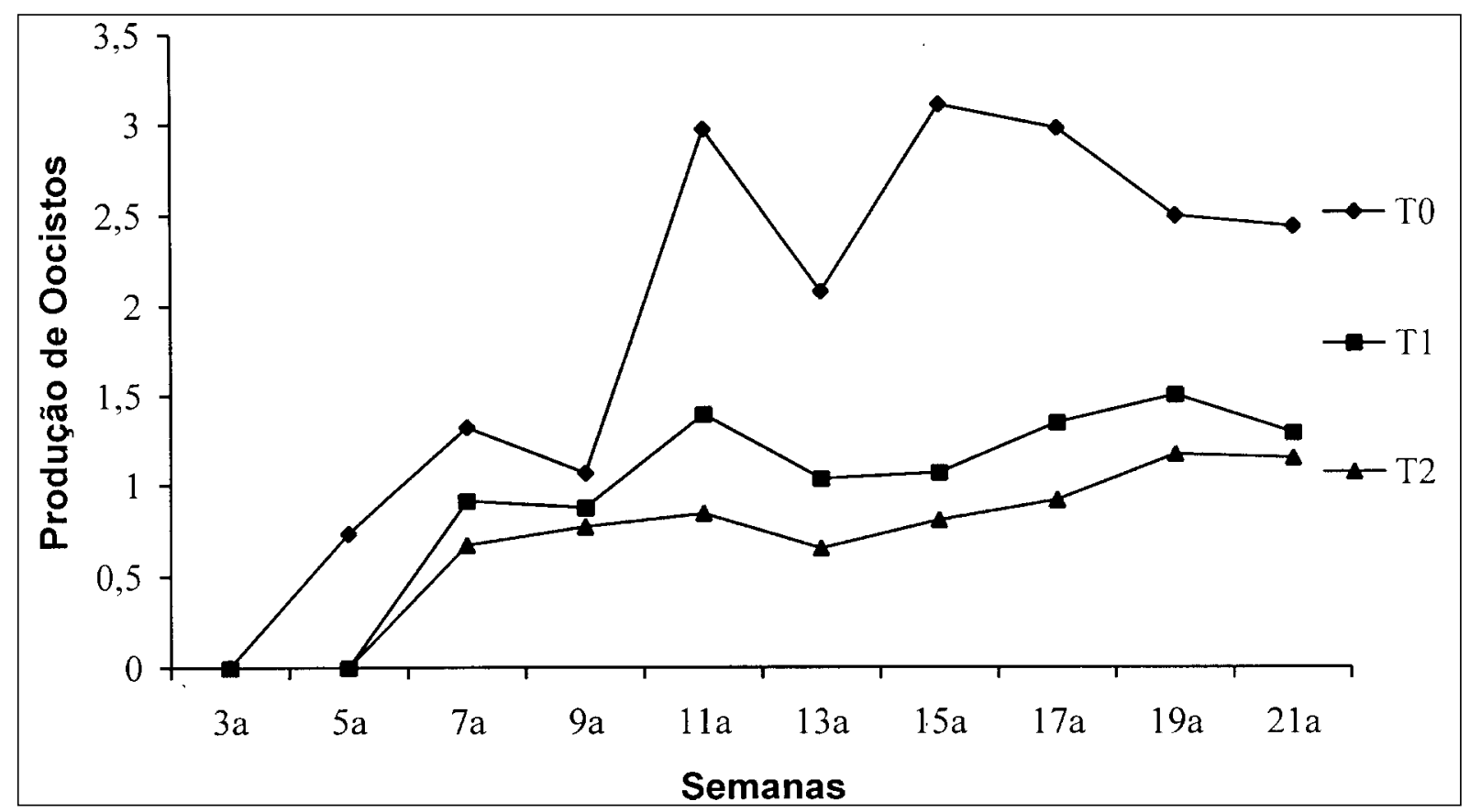

Figura 1 - Número médio de oocistos por grama de fezes (OOPG) ( $( \pm$ erro padrão) em caprinos submetidos aos tratamentos preventivos com salinomicina, nas fases de cria e recria. Dados transformados para $\log (\mathrm{OOPG}$ x $0,02+4,5)$. T0= Grupo não medicado (controle); $\mathrm{T} 1$ e $\mathrm{T} 2=$ medicados com 1 e $2 \mathrm{mg} / \mathrm{kg}$, respectivamente. 
terceiro mês de vida, faixa etária na qual geralmente ocorrem os picos de eliminação de oocistos de Eimeria nas fezes (LIMA et al., 1985), não foi observado sintomatologia clínica de eimeriose.

O rendimento de carcaça e a massa corporal também foram influenciados pelo tratamento preventivo para Eimeria com o uso da salinomicina. Ambos apresentaram comportamento linear positivo e significativo $(\mathrm{P}<0,05)$. Os incrementos foram de $1,34 \mathrm{e}$ 1,15 unidades de rendimento de carcaça e massa corporal, respectivamente, para cada unidade de salinomicina consumida. As correlações de -0,50 e 0,46 entre rendimento de carcaça e massa corporal com oocistograma na fase de recria foram significativas $(\mathrm{P}<0,01)$, confirmando que a adição de salinomicina nas doses de $1 \mathrm{mg} / \mathrm{kg}$ e $2 \mathrm{mg} / \mathrm{kg}$ à dieta foi efetiva em melhorar o desempenho dos animais.

$O$ fato de se ter verificado um comportamento linear significativo $(\mathrm{p}<0,01)$ para a maioria das variáveis estudadas indica que as doses de salinomicina utilizadas (1 e $2 \mathrm{mg} / \mathrm{kg}$ ) não foram suficientes para detectar a partir de que ponto ocorreu a estabilização da linearidade e o decréscimo ou acréscimo da curva, o que permitiria determinar os pontos de mínimo ou de máximo. No entanto, considerando tratar-se de um produto tóxico (SANTA ROSA \& PINHEIRO, 1996), cuja DL-50 para caprinos ainda é desconhecida, doses mais elevadas poderiam implicar intoxicação dos animais. No presente trabalho, não foram observados sinais clínicos de intoxicação e mortalidade pela salinomicina na dose de $2 \mathrm{mg} / \mathrm{Kg}$, conforme registrado por SANTA ROSA \& PINHEIRO (1996). No trabalho realizado por estes autores, a salinomicina foi adicionada ao leite oferecido ao grupo, duas vezes por dia (manhã e tarde). Como existem animais que ingerem uma quantidade de leite maior que outros, é possí- vel que aqueles que apresentaram sintomas de intoxicação e mortalidade, tenham ingerido uma dose superior a $2 \mathrm{mg} / \mathrm{kg}$ de salinomicina. No presente trabalho, a salinomicina foi administrada individualmente, o que assegurou a ingestão de exatamente $2 \mathrm{mg} / \mathrm{Kg}$ de salinomicina, indicando que esta dose não é tóxica para os caprinos.

A margem bruta, calculada pela diferença entre a receita bruta e os custos variáveis (NEVES \& SHIROTA, 1986), está representada na tabela 3. O aumento na dosagem da salinomicina de $1 \mathrm{mg} / \mathrm{kg}$ para $2 \mathrm{mg}$ / $\mathrm{kg}$, elevou o custo total, embora também tenha ocorrido um aumento da receita do $\mathrm{T} 1$ para o T2, impulsionado pelo crescimento no ganho de peso (Tabela 3).

Entretanto, embora não tenha sido observado diferença significativa ( $p>0,05)$ entre os valores da margem bruta dos níveis testados, observa-se que o aumento de consumo da salinomicina proporcionou maior desenvolvimento ponderal dos animais, elevando a receita e, consequentemente, a margem bruta. Além do aspecto econômico a redução no oocistograma dos animais é indicativo de melhor saúde destes, fator de elevada importância especialmente para animais jovens.

\section{CONCLUSÕES}

De acordo com os resultados obtidos no presente trabalho, conclui-se que o tratamento preventivo com a salinomicina é eficaz para o controle da eimeriose de caprinos leiteiros, desde que a medicação preventiva seja administrada aos cabritos na dose de $1,0 \mathrm{mg} / \mathrm{kg}$, a partir da segunda semana de vida. $\mathrm{O}$ tratamento preventivo, quando iniciado na fase de cria, reduz significativamente a infecção dos cabritos por Eimeria durante a fase de recria, promovendo um melhor ganho de peso.

Tabela 3 - Estrutura de custo, receita e a margem bruta da produção de cabritos, realizando controle preventivo da eimeriose.

\begin{tabular}{lccc}
\hline & & Níveis de utilização \\
Discriminação & T0 (controle) & T1 (1mg/kg) & T2 (2mg/kg) \\
\hline Receita (R\$/cab.) & 14,80 & 16,48 & 18,23 \\
- Ganho médio de peso (kg/cab) & 11,39 & 12,68 & 14,02 \\
Custo (R\$cab.) & 11,65 & 13,56 & 14,55 \\
- Leite & 2,18 & 2,00 & 2,18 \\
- Concentrado & 3,13 & 3,62 & 3,71 \\
- Volumoso & 1,68 & 2,53 & 2,30 \\
- Salinomicina & - & 0,75 & 1,70 \\
- Vacina (febre aftosa) & 0,35 & 0,35 & 0,35 \\
- Vermífugo & 0,33 & 0,33 & 0,33 \\
- Mão-de-obra & 3,98 & 3,98 & 3,98 \\
Margem bruta (R $\$$ cab.) & 3,15 & 2,93 & 3,68 \\
\hline
\end{tabular}




\section{REFRÊNCIAS BIBLIOGRÁFICAS}

AUGUSTINE, P.C. et al. Effect of inophorus anticoccidials on invasion and development of Eimeria: comparasion of sensitive and resistant isolates and correlation with drug up take. Poultry Science, v.66, p.960-965, 1987.

FACURY FILHO, E.J. Evolução da infecção por Eimeria spp., em bezerros naturalmente infectados e seu controle através da administração de anticoccidiostáticos no suplemento mineral. 1992. 99f. Dissertação (Mestrado, em Medicina Veterinária Preventiva) - Curso de Pós-graduação em Ciência Animal, Escola de Veterinária, UFMG.

LIMA, J. D. et al. Control of bovine occidiosis with aprolium and monesin under brasilian husbandry conditions. In: WORD ASSOCIATION FOR THE ADVANCEMENT OF VETERINARY PARASITOLOGY, 11., 1985, Rio de Janeiro. Abstracts... Rio de Janeiro, 1985. p.35.

NEVES, E.M.; SHIROTA, R. Condições sobre a importância, determinação e atualização dos custos agrícolas. Revista da ADEALQ, Piracicaba, n.4, p.62-69, 1986.

OLIVEIRA, P.R. et al. Controle da infecção por Eimeria spp., em caprinos pela administração contínua de salinomicina no suplemento mineral. Arquivo Brasileiro de Medicina Veterinária e Zootecnia, Belo Horizonte, v.49, n.3, p.291-296, 1997.

PIMENTEL GOMES, F. Curso de estatística experimental. 7.ed. Piracicaba : Nobel, 1977, p.38-41.

REBOUÇAS, M.M. et al. Identificação de espécies do gênero Eimeria Schneider, 1985, parasitas de caprinos no Estado de São Paulo, Brazil (Apicomplexa: Eimeriidae). Revista Brasileira de Parasitologia Veterinária, São Paulo, v.1, n.1, p.6164, 1992.

SANTA ROSA, J.; PINHEIRO, P. A. Intoxicação por salinomicina em cabritos. In: CONGRESSO BRASILEIRO DE MEDICINA VETERINÁRIA, 24., 1996, Goiânia. R esumos... Goiânia, 1996. p.60.

SUAREZ, I.R.H. Avaliação das parasitoses gastrointestinais em uma exploração intensiva de caprinos leiteiros. 1995. 134f. Dissertação (Mestrado em Veterinária Preventiva) - Curso de Pós-graduação em Ciência Animal, Escola de Veterinária, UFMG.

UENO, H.; GONÇALVES. P.C. Manual para o diagnóstico das helmintoses de ruminantes. 2.ed. T okyo, Japão : Universidade Autônomas de Santo Domingo, 1988. 166p. 